\title{
Polyphenolics compound variation in foxtail millet (Setaria italica) germplasm and establish a core collection
}

\author{
Lee Myung Chul*, Choi Yu-Mi, Yoon Hyemyeong, Lee Sukueung, \\ Yoon-Hyun Do, Oh Sejong \\ National Agrobiodiversity Center, National Institute of Agricultural Sciences, RDA, Republic of Korea \\ *e-mail:mcleekor@korea.kr
}

Setaria italica (L.) P. Beauv. is one of the most widely cultivated species of millet in the Korea and have considerable attention due to its nutritional quality related to high content of dietary fiber, protein, starch patterns and high level of minerals. This research was aimed to study variation of polyphenols compound in foxtail millet germplasm and combined it with basic agronomic traits and molecular markers to establish a core collection. Total phenolic content ranged from 11 to $87 \mathrm{mg}$ gallic acid equiv (GAE)/100g and antioxidant activity was showed from $3.35051 \%$ by DPPH scavenging activity. Here we assessed the genetic diversity and population structure in a large germplasm collection of 785 accessions by employing EST-SSR markers, morphological traits he phenolic content and antioxidant activity. The germplasm collection was separated into three groups based on population structure analysis, whereas principal coordinate analysis (PCoA) could not cluster accessions according to their geographic origin. Subsequently, a core collection with a total of 170 accessions $(21.66 \%)$ was selected from the whole set of germplasm by combining allelic variations of 22 EST-SSR markers and their traits. The core collection optimally represented the whole germplasm collection and displayed a similar level of genetic diversity, population structure, and phenotypic variations based on various genetic analyses such as Shannon-Weaver and Nei's diversity indices and PCoA, while phenotypic traits were analyzed by mean, range, and principal component analysis. This core collection of foxtail millet will be a primary resource for further genetic analysis and development of appropriate. 